\title{
Effects of Sample Preparation Technique on Quantitative Analysis of Automotive Fuel Cell Catalyst Layers
}

\author{
Lis G. de A. Melo ${ }^{1}$, Vincent Lee ${ }^{2}$, Darija Susac ${ }^{3}$, Viatcheslav Berejnov ${ }^{3}$ Juergen Stumper $^{3}$, Gianluigi A. Botton ${ }^{1}$ \\ and Adam P. Hitchcock ${ }^{2}$ \\ 1. Department of Material Science and Engineering - McMaster University, Hamilton, ON. Canada \\ ${ }^{2}$ Department of Chemistry and Chemical Biology - McMaster University, Hamilton, ON. Canada \\ ${ }^{3}$ AFCC Automotive Fuel Cell Cooperation Corp., 9000 Glenlyon Parkway, Burnaby, BC, Canada
}

Environmental concerns about carbon emissions are motivating development of Proton-Exchange Membrane Fuel Cell (PEM-FC) technology for automotive propulsion. Catalyst Coated Membranes (CCM), which are the electrochemical 'heart' of PEM-FC, consist of a proton-conductive polymer membrane embedded between two porous, electron conductive electrodes. The catalyst in the cathode consists of Pt nanoparticles on a carbon support which are coated with a proton-conductive perfluorosulfonic acid (PFSA) ionomer [1]. The oxygen reduction reaction occurs at the triple junction of these three components. One aspect needing improvement to achieve successful commercialization is a reduction of the rate of degradation of catalyst components with electrochemical cycling. Different microscopy techniques are helping optimize the system to improve durability of PEM-FC. However, sample preparation can affect the results of the microanalysis. In order to fully understand each component and how it degrades, it is important to understand and eliminate sample preparation artifacts.

Two sample preparation techniques were applied to the same CCM sample: ultra-microtoming and Focused Ion Beam (FIB) milling (Zeiss Nvision40 FIB operated at $30 \mathrm{kV}$, with final polishing at $10 \mathrm{kV}, 80 \mathrm{pA}$ ). Although ultra-microtoming is widely used to prepare PEM-FC samples, a major disadvantage is epoxy penetration into the porous structure of the carbon support, which makes PFSA visualization challenging in transmission electron microscopy (TEM) unless staining is applied [2]. This has lead to an alternate sample preparation involving sandwiching the CCM between two softened beads of polystyrene (PS) aiming to minimize penetration of embedding material into the catalyst layer. Although initial TEM images suggested that the polystyrene did not significantly penetrate into the catalyst layer, Scanning Transmission X-ray Microscopy (STXM) demonstrated otherwise (Fig. 1). FIB sectioning is an alternative sample preparation technique which does not introduce additional carbon-containing components, and thus is commonly used in combination with scanning electron microscopy (SEM) to study cathode porosity and to prepare samples with uniform thickness for TEM analysis. While FIB samples are great for porosity analysis, we have demonstrated recently that there are associated artifacts such as Ga contamination and carbon-structure amorphization [3].

STXM has proven useful for analyzing structural damage caused by FIB to soft materials [4]. A comparison of STXM-derived C 1s spectra of the cathode region of the same CCM sample prepared by ultramicrotoming and FIB (Fig. 2) shows that FIB causes a loss of the $\mathrm{C}$ 1s fine structure and broadening of the $\mathrm{C}$ 1s continuum, likely induced by amorphization of both the graphitic carbon support and the PFSA. A STXM F 1s image sequence taken from both samples also shows extensive spectral modification of the $\mathrm{F} 1 \mathrm{~s}$ signal, indicating structural damage to the PFSA (Fig. 3). In order to quantity the fluorine content in both samples, internal reference spectra for the PFSA were obtained from the membrane of the microtome sample and the whole area of the FIB sample. These spectra were set to quantitative intensity scales (optical density per $\mathrm{nm}$ ) by scaling to match the elemental response for PFSA. A pixel-by-pixel fit to the F 1s image sequence yielded quantitative component maps for the PFSA and non-fluorinated components. Since the FIB and microtomed samples have different thicknesses $(70 \pm 15$ $\mathrm{nm}$ and $53 \pm 8 \mathrm{~nm}$, respectively, estimated from the C 1s stacks), the fractional amount of PFSA was calculated by dividing the PFSA component map by the sum of the PFSA and non-fluorinated maps. This analysis shows that FIB not only damages the structure of PFSA in the cathode layer (as indicated by the shape change), but it is also responsible for considerable mass loss (Fig. 3b). While STXM measurements of additional FIB samples are needed for a statistically unchallengeable result, the two FIB sections analyzed so far have similar results [5]. 
[1] F. Barbir in "PEM Fuel cells", 2005 (Elsevier, USA) p.

$1-16$.

[2] H. Uchida et al, J. Phys. Chem. Let. B, 110 (2006) p.13319.

[3] L.G.A. Melo et.al ECS Transactions 58 (2013) 251.

[4] N. D. Bassin et al, J. of Microscopy, 245 (Pt. 3) (2011) p. 288

[5] Research funded by the CARPE-FC network. We thank Julia Huang and Marcia West for sample preparation. TEM was performed at the CCEM, which is supported by NSERC and McMaster University. The STXM work was performed at the Canadian Light Source, beamline 10ID-1 SM. CLS is supported by NSERC,CIHR, NRC

and U. Saskatchewan.

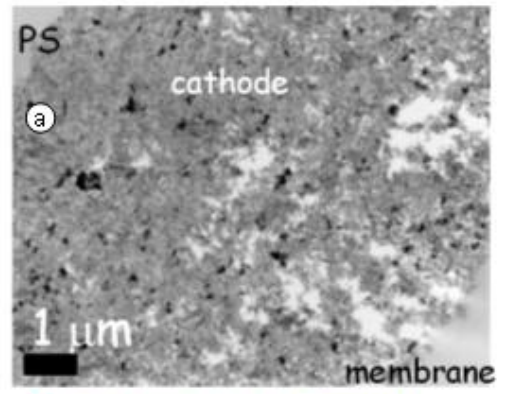

PS membrane (PFSA) C_support

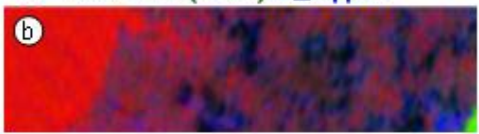

with PFSA in cathode enhanced ${ }^{*} 3$

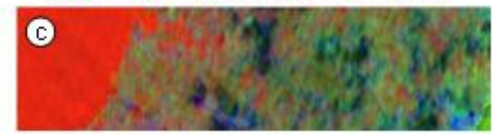

Fig. 1 (a) Bright field TEM of microtomed CCM indicating the PS, cathode and membrane $(\mathbf{b}, \mathbf{c})$ color composites of PS, PFSA and C_support maps derived from a STXM C Is image sequence.

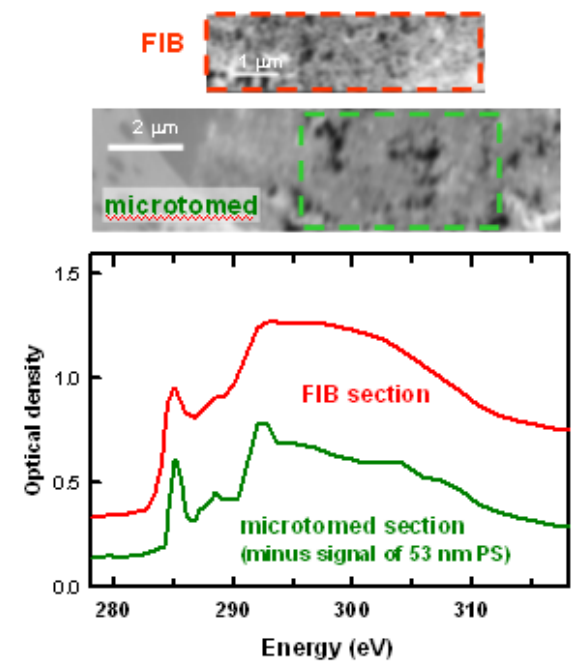

Fig. 2 C 1s X-ray absorption spectra of the cathode of the same CCM sample prepared by FIB (red) and ultramicrotome (green). The dotted rectangles on the images (sum of all STXM images in the C 1s sequence) indicate the area used for the spectra. The PS signal in the microtomed spectrum has been subtracted.
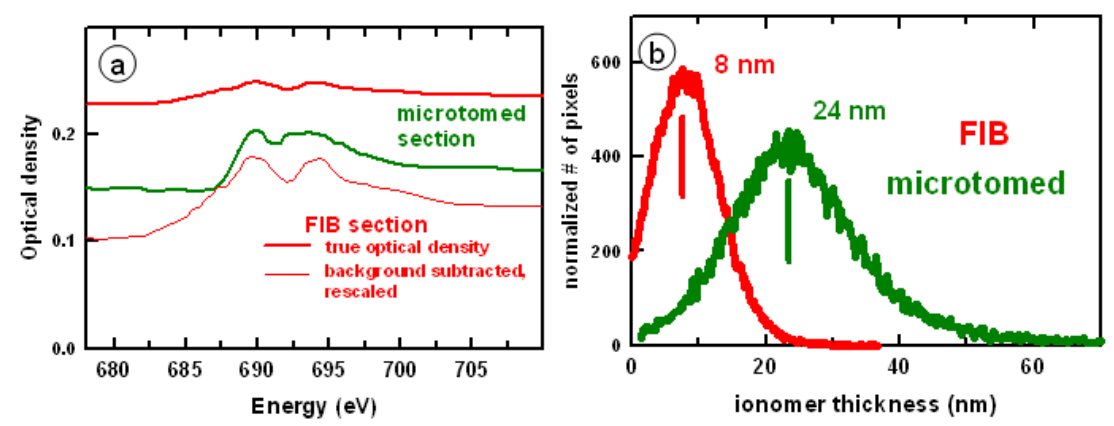

Fig. 3 a) STXM-derived F 1s spectra of the cathode of microtome and FIB samples. Both the shape and magnitude are dramatically different. b) Histograms of thickness of ionomer (PFSA) in the cathode in microtome and FIB samples determined from F 1s analysis. Estimated average fractional amounts are 0.5 and 0.1 respectively. 